\title{
Avaliação de Interferentes Externos na Análise de Petróleos e suas Frações por Espectroscopia no Infravermelho Médio
}

\author{
Victor G. K. S. Cardoso, Laine B. Pereira \& Paulo R. Filgueiras
}

A minimização de erros é de grande interesse nas análises químicas. Assim, este estudo investigou a diferença entre duas metodologias de obtenção de espectros de infravermelho médio, eliminando ou não o contato de amostra com a radiação externa. Foram obtidos espectros em quintuplicata de dez amostras diferentes de petróleo e suas frações para cada uma das metodologias. Através da PCA foi possível discriminar as duas técnicas. A metodologia já utilizada apresentou uma maior concordância dos dados e a metodologia proposta apresentou uma grande variabilidade dos espectros.

Palavras Chave: Infravermelho Médio; Interferentes; Petróleo; Química Analitica; Quimiometria; PCA.

Minimizing errors is deeply important in chemical analysis. Thus, this study investigated the difference between two methods of obtaining mid-infrared spectra, eliminating or not the sample contact with the external radiation. The spectra were obtained fivefolf from ten different petroleum samples and their fractions for each of the methodologies. Through the PCA was possible to discriminate these techniques. The methodology already used presented a better agreement of the data and the proposed methodology presented a great variability of the spectra.

Keywords: Mid-infrared; Interferers; Petroleum; Analitical Chemistry; Chemometrics, $P C A$. 


\section{Introdução}

O petróleo é uma mistura complexa de compostos orgânicos, podendo ser decomposto em quatro classes de componentes: hidrocarbonetos saturados, aromáticos, resinas e asfaltenos. A composição química de cada óleo varia de acordo com a matriz geradora e toda a história geológica da formação, sendo possível uma variedade infinita de óleos. ${ }^{1} \mathrm{Na}$ avaliação do perfil de cada óleo é realizada sua caracterização físico-química, sendo fundamental para todo o processo industrial, desde a extração até o refino e o abastecimento, além da importância econômica e ambiental, no tratamento dos resíduos oleosos. Para isso, diversas características são avaliadas, como a densidade, grau API, viscosidade, acidez, salinidade, teores de nitrogênio e enxofre. ${ }^{2}$

Os métodos tradicionais para caracterização físicoquímica completa de um óleo bruto e seus derivados são bastante laboriosos, demandando muito tempo e recursos financeiros, pois para uma avaliação completa do óleo pode ser necessária a realização de até 700 ensaios. Diante disso, é importante para a industrial petrolífera o desenvolvimento de novas técnicas que garantam um bom resultado, rapidez e baixo custo. ${ }^{3,4}$

Como alternativa aos métodos tradicionais, a associação da quimiometria e técnicas espectroscópicas, como o infravermelho, tem apresentado um grande potencial na caracterização físico-química de óleos brutos, pois podem fornecer uma grande quantidade de informação em questão de segundos. O infravermelho médio é uma técnica eficiente para identificar grupos funcionais orgânicos, mostrando muita eficiência na análise de petróleo. 4,5

Com a utilização de novas técnicas é extremamente importante otimizar a técnica para gerar o melhor sinal possível para a matriz oleosa a ser investigada. Sabendo que o erro é algo inerente aos ensaios, sendo impossível realizar uma análise química livre de erros e incertezas, faz-se necessário buscar formas de minimizá-los de modo a afetar cada vez menos nas análises realizadas, pois uma análise ruim ou que não represente a amostra pode trazer muito prejuízo. ${ }^{6}$

Diante do exposto, este trabalho tem como objetivo investigar a ação de interferentes externos na atual metodologia da análise por infravermelho médio de petróleo bruto e seus derivados.

\section{Metodologia}

Para a realização do trabalho foram utilizadas dez amostras de petróleo bruto e algumas frações oleosas organizadas de acordo com a seguinte legenda: (a) petróleo pesado, (b) petróleo pesado, (c) lubrificante, (d) gás condensado, (e) gasolina comercia comum, (f) diesel S-10, (g) diesel fração antiga, (h) diesel fração nova, (i) querosene e (j) nafta. Para a obtenção dos espectros foi utilizado o espectrômetro Cary 630 FTIR da Agilent Technologies ${ }^{\circledR}$, que é um equipamento de infravermelho operado no modo transflectância. Este equipamento possui um aparato que cobre a amostra eliminando os efeitos da luz externa e da atmosfera, sendo o mesmo indicado para análise de amostras sólidas. O trabalho consistiu em verificar a utilização desse aparato ao analisar amostras líquidas. Para isso, para cada amostra foram obtidos cinco espectros com o aparato aberto e mais cinco espectros com o aparato fechado, totalizando dez espectros por amostra, num total de cem espectros, onde os mesmos foram obtidos de forma aleatória. A etapa posterior consistiu no tratamento quimiométrico, utilizando um modelo de análise por componentes principais (PCA), de modo a verificar se existe diferença entre as metodologias e qual seria a mais adequada.

\section{Resultados e Discussão}

O trabalho foi realizado no objetivo de otimizar a metodologia já aplicada nas análises petróleo e suas frações por espectroscopia no infravermelho médio. Para isso, foram escolhidas várias amostras de diferentes frações do petróleo para testar o efeito da proposta sob as diferentes matrizes oleosas. Os espectros podem ser classificados de duas formas diferentes, a primeira forma é sobre a utilização do aparato, que por sua vez é divido em aberto e fechado. A segunda forma é sobre a amostra, podendo ser divido em dez amostras diferentes. O perfil dos espectros pode ser observado na Figura 1. Os espectros na cor azul, metodologia atual, apresentam menor variação na linha de base espectral. 

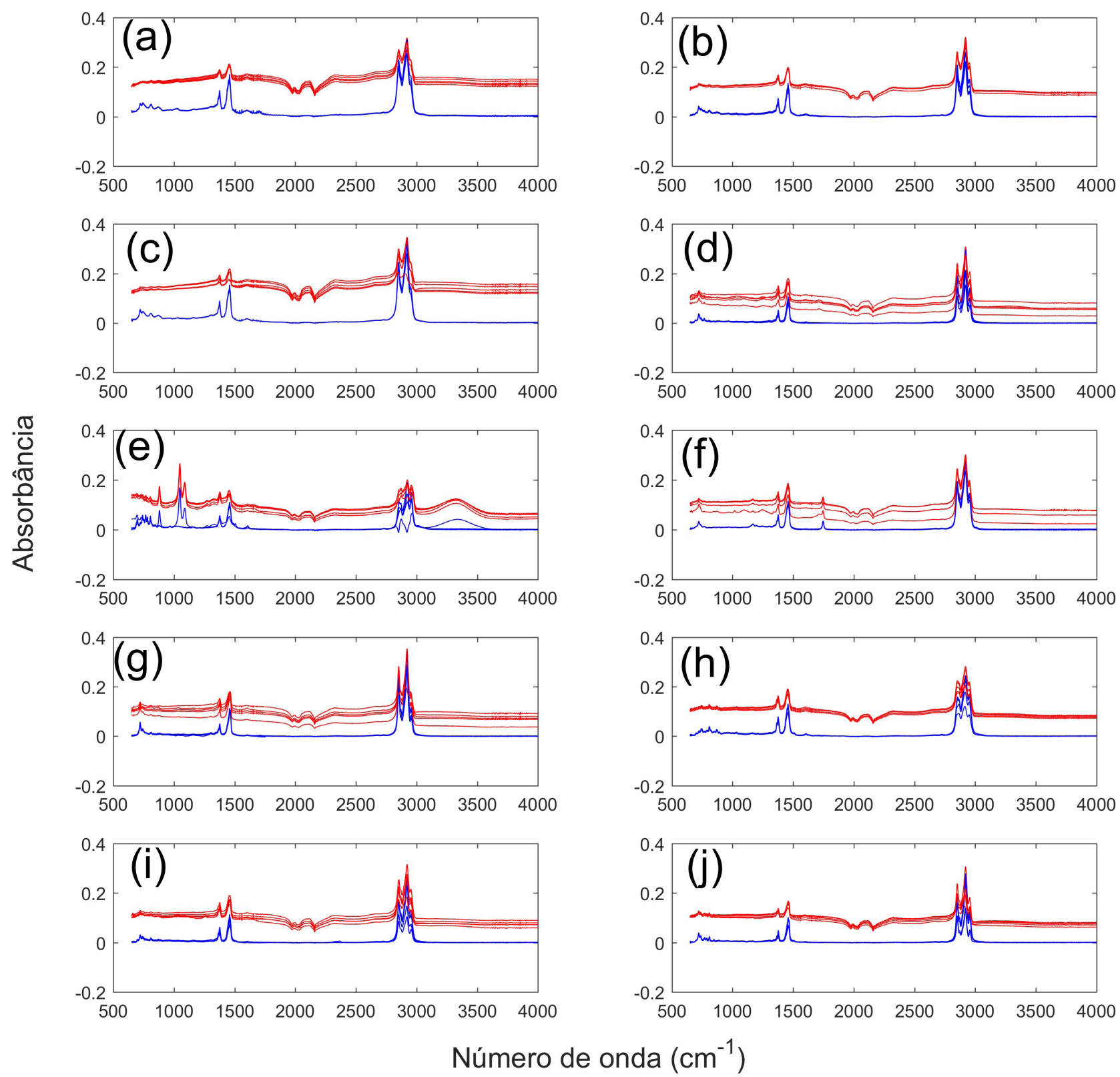

Figura 1. Espectros de infravermelho médio para as amostras de (a) petróleo pesado, (b) petróleo pesado, (c) lubrificante, (d) gás condensado, (e) gasolina comercia comum, (f) diesel S-10, (g) diesel antiga, (h) diesel nova, (i) querosene e (j) nafta. O espectro azul apresenta a metodologia atual, mantendo o aparato aberto, enquanto o espectro vermelho apresenta a metodologia proposta, mantendo o aparato fechado. 
Para uma melhor interpretação das informações e uma avaliação minuciosa dos dados, foi aplicada a análise por componentes principais (PCA). As duas primeiras componentes principais explicaram $98,25 \%$ da variância total dos dados originais. Essas informações podem ser observadas na Figura 2.

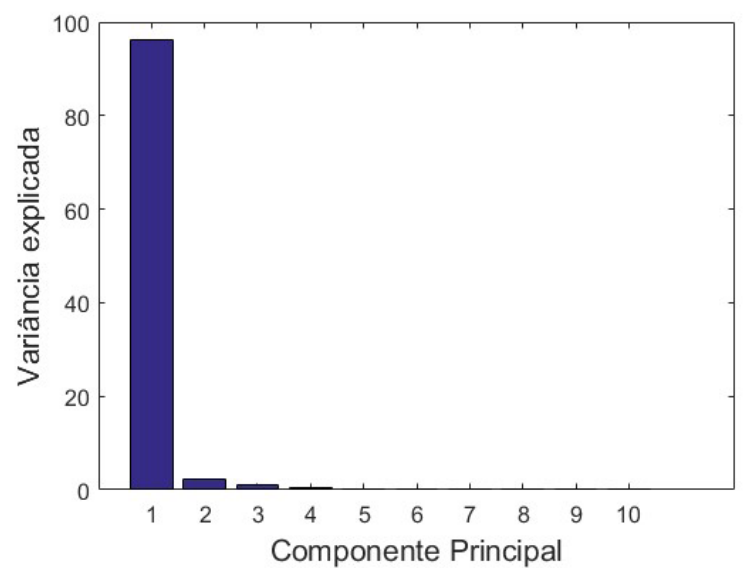

Figura 2. Quantidade de variância encontrada em cada componente principal.

A projeção das amostras no subespaço formado pelas duas primeiras componentes (Figura 3) mostra um comportamento muito diferente para os dois tipos de ensaios (Fechado e Aberto). Verificou-se que na metodologia atual uma variabilidade muito menor quando comparadas às análises da metodologia proposta.
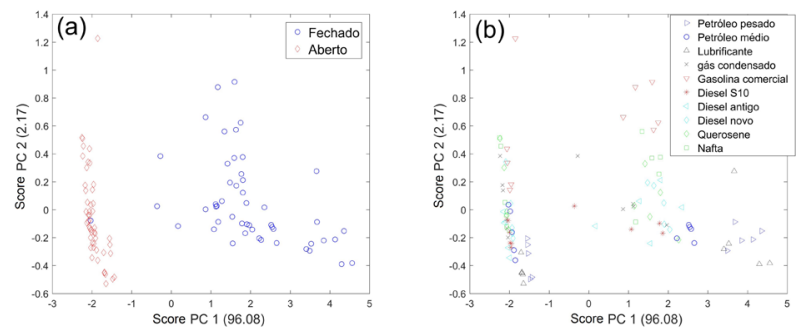

Figura 3. Gráfico dos scores PC1 x PC2 correspondentes às amostras utilizadas.
Uma explicação para tal acontecimento pode estar na obtenção do background. Em todos os ensaios antes de obter o espectro da amostra é coletado um background do ar atmosférico, que é realizada para ser subtraído do espectro da amostra. Quando a amostra líquida é fechada um menor caminho óptico para a radiação é encontrado, logo ocorre a subtração do background de uma amostra que não está em contato com o ar atmosférico. Isso pode ser corroborado através da absorbância negativa por volta de 2000 e 2200 cm-1, região de absorção do $\mathrm{CO}_{2}$.

$\mathrm{O}$ efeito não ocorre nas amostras sólidas, pois o procedimento evita o espalhamento da radiação, além de haver ar atmosférico entre as minúsculas partículas dos sólidos.

\section{Conclusões}

Por meio do estudo, constatou-se que a metodologia já aplicada, utilizando o aparato aberto, foi considerada melhor por apresentar uma menor variabilidade nos espectros quando comparada à metodologia proposta, utilizando o aparato fechado. Também foi possível observar que a coleta do background do espectro já é suficiente para minimizar a ação dos possíveis interferentes devido à radiação externa e o ar atmosférico durante a análise.

\section{Agradecimentos}

Primeiramente agradeço a Deus. Agradeço à UFES e ao LabPetro por terem permitido a realização dos trabalhos.

\section{Referências Bibliográficas}

1. Thomas, J. E. Fundamentos da engenharia do petróleo. Rio de Janeiro: Editora Interciência, 2001. 271 p.

2. Filgueiras, P. R. Determinação da composição de blends de petróleos utilizando FTIR-ATR e calibração multivariada. 2011. $113 \mathrm{f}$.

Dissertação (Mestrado em Química). Departamento de Química, Universidade Federal do Espírito Santo, Vitória, 2011.

3. Dias, J. C. M. Estudo exploratório das propriedades de caracterização de petróleos brasileiros. Dissertação de Mestrado. Departamento de Química da Universidade Federal do Rio de Janeiro, Rio de Janeiro, 2005. 
4. Rainha, K. P. Estudo de alguns parâmetros físico-químicos de petróleos utilizando modelagem multivariada associada a espectroscopia no infravermelho. Dissertação de Mestrado.

Departamento de Química, Universidade Federal do Espírito Santo, Vitória, 2014.

5. Pasquini, C. Near Infrared Spectroscopy: fundamentals, practical aspects and analytical applications. Journal of the Brazilian Chemical Society, 14, 198-219, 2003.

6. Skoog, D. A., West, D. M.; Holler, F.J.; Crouch, S. R. Fundamentos de Química Analítica. 8a. São Paulo: Cengage Learning, 2006. 1000 p.

\section{Victor G. K. S. Cardoso ${ }^{1 *}$, Laine B. Pereira ${ }^{2} \&$ Paulo R. Filgueiras $^{\top}$}

\footnotetext{
${ }^{1}$ Departamento de Química, Centro de Ciências Exatas, Universidade Federal do Espírito Santo - UFES. Avenida Fernando Ferrari, 514 Goiabeiras - 29075-910, Vitória - ES, Brasil.

${ }^{2}$ Programa de Pós-graduação em Química, Centro de Ciências Exatas, Universidade Federal do Espírito Santo - UFES. Avenida Fernando Ferrari, 514 - Goiabeiras - 29075-910, Vitória - ES, Brasil.
}

*E-mail: victor.cardoso@aluno.ufes.br 